\title{
Levels of DKK1 in patients with acute myocardial infarction and response to atorvastatin
}

\author{
José Luis Pérez Castrillón ${ }^{\mathrm{a}, \mathrm{d}, *}$, Angel San Miguel ${ }^{\mathrm{a}}$, Gemma Vega ${ }^{\mathrm{a}}$, Laura Abad ${ }^{\mathrm{a}}$, María Andres Domingo ${ }^{\mathrm{b}}$, \\ Manuel Gonzalez Sagredo ${ }^{b}$, Daniel de Luis ${ }^{b, d}$, Antonio Duenas-Laita ${ }^{c}$
}

a Internal Medicine Department, Rio Hortega University Hospital, Faculty of Medicine, Valladolid, Spain

${ }^{\mathrm{b}}$ Institute of Endocrinology and Nutrition Research Support Unit, Rio Hortega University Hospital, Faculty of Medicine, Valladolid, Spain

c Clinical Pharmacology Unit, Río Hortega University Hospital, Valladolid, Spain

${ }^{\mathrm{d}}$ RETICEF, Spain

A R T I C L E I N F O

Article history:

Received 9 July 2009

Accepted 25 July 2009

Available online 31 August 2009

Keywords:

Acute myocardial infarction

Atorvastatin

DKK1

Osteoporosis

The atherosclerosis that appears in coronary, cerebrovascular and peripheral arterial disease is responsible for most cardiovascular diseases. It is characterized by chronic arterial inflammation caused and exacerbated by disorders of the lipidic metabolism and other clearly identified risk factors [1]. Calcification, which is initiated by an active process in which inflammatory cytokines and other mediators that regulate the phospho-calcium metabolism intervene, is characteristic of atherosclerosis [2]. These mechanisms can intervene in an opposite phenomenon that takes place at the level of the bone characterized by a reduction in bone mineral content and alterations in the microarchitecture that define osteoporosis. The association between the two diseases, which share mechanisms but have a different expression, is noteworthy.

The Wnt-LPR5 signalling pathway plays an important role in skeletal homeostasis, especially in regulating osteoblastic activity. It is formed by a series of elements, WnT ligands, and a receptor complex, which is constituted by the Frizzled protein and LPR5-6 [3]. Recently, a missense mutation in LPR6 that codifies a coreceptor has been described in an Iranian family. Cysteine is replaced by arginine, damaging in vitro Wnt signalling. These patients have a greater risk of coronary disease, low bone mineral density (BMD) and osteoporotic fracture, suggesting that the two diseases may be pleiotropic consequences of the alteration of the Wnt signalling pathway [4].

Wnt signalling pathway activation is regulated by various inhibitors, including DKK1, which interacts with a transmembrane protein, kremen, to prevent the activation of the LPR5 coreceptor, leading to internalization of the DKK/LPR complex with a loss of the Wnt signal [5]. Studies in experimental animals have shown a possible role of DKK1 in the regulation of bone homeostasis, although there are few studies in humans and in patients with acute coronary syndrome.

The objective of this study was to evaluate DKK1 levels in patients with acute myocardial infarction, the response to atorvastatin and the relationship with bone mass.

Patients with acute myocardial infarction were included. Exclusion criteria were chronic alcohol abuse, neoplasia, chronic renal failure, hyper- and hypocalcaemia, hyperparathyroidism and the use of drugs

* Corresponding author. Hospital Río Hortega Cardenal Torquemada s/n 47010

Valladolid, Spain. Tel.: +34983 420400; fax: +34983 331566.

E-mail address: castrv@terra.es (J.L. Pérez Castrillón). modifying BMD. Patients were allocated to low $(10-20 \mathrm{mg})$ and high doses $(40-80 \mathrm{mg}$ ) of atorvastatin according to baseline levels of cholesterol and triglycerides and the index of vascular risk. Measurements were made at baseline and at 12 months of follow up. The control group was patients of the same age and sex without coronary disease.

Blood samples were obtained after 8 fasting hours. DKK1 was determined by immunoassay (Biomedica, Wien, Austria) with a $9 \%$ interassay coefficient of variation. Densitometric studies were conducted in the lumbar spine (L2-L4) and femoral neck and trochanter using an X-ray densitometer (DXA, Lunar Corporation, Madison, Wisconsin, USA).

The results are expressed as mean \pm standard deviation. All variables were analyzed using descriptive statistics. Comparisons of the mean were made using the paired $t$-test and the Mann-Whitney nonparametric $U$ test. The statistical analysis used SPSS (SPSS, Chicago, Ill; Base 11.4 for Windows) Twenty-one patients with acute coronary syndrome and twenty-three controls with a mean age of $61 \pm 9$ years were included. Patients had higher levels of DKK1 than controls, (111 \pm $41 \mathrm{nmol} / \mathrm{l}$ versus $84 \pm 27 \mathrm{nmol} / \mathrm{l}, p=0.014)$. The prevalence of osteoporosis ( $T$ score $<-2.5$ in the lumbar spine and/or hip) was $20 \%$. There were significantly significant differences in DKK1 in patients with or without osteoporosis, $(137.5 \pm 33 \mathrm{nmol} / \mathrm{l}$ versus $95.4 \pm 36 \mathrm{nmol} / \mathrm{l}$ $p=0.021)$. Analysis of the response to atorvastatin showed reduced DKK1 levels without differences between patients and controls, $(90 \pm$ $39 \mathrm{nmol} / \mathrm{l}$ versus $84 \pm 27 \mathrm{nmol} / \mathrm{l}, p=0.551$ ). There were no differences in the response of bone mass to atorvastatin according to the drug dose.

The patients with acute coronary syndrome had high levels of DKK1 than the control group. In addition, when patients were divided into osteoporotic and nonosteoporotic groups, DKK1 differed significantly. The effect of atorvastatin showed a significant reduction at 12 months of follow up compared with baseline levels.

Ueland et al. [6] study the levels of DKK1 in patients with stable angina, unstable angina and healthy control subjects. Patients who are stable and particularly those who are unstable had markedly raised serum levels of DKK1, compared with controls. Our results are similar. There are no studies analyzing DKK1 levels and bone mineral density in patients with acute coronary syndrome, although one study found that patients with Wnt pathway polymorphisms had early ischemic heart disease and a greater risk of fractures [4]. In experimental animals, increased calcification of the aortic valve and arteries after increasing the production of $\beta$-catenin by activation of the Wnt pathway was observed [7]. Our study suggests the possible participation of DKK1 in osteoporosis associated with acute coronary syndrome.

Experimental, but not human, studies have analyzed the effect of atorvastatin on the $\beta$-catenin signalling pathway. Atorvastatin decreases the expression of LRP5 with a reduction in $\beta$-catenin concentrations in calcified aortic valves in rabbits, inhibiting calcification [7]. At the skeletal level, the behaviour is inverse: a reduction in DKK1 levels can activate the Wnt signalling pathway and increase bone formation [8]. 
Experimental studies show the mechanisms used by statins to act on both components of bone remodelling, formation and resorption, and demonstrate a beneficial effect on bone balance. Not all studies conducted on humans reflect these data, which is probably due to methodological differences and the statin dose. Our study describes a possible new anabolic mechanism of action of atorvastatin at the level of the bone, stimulation of the Wnt pathway through a reduction in DKK1 levels which increases the number and activity of osteoblasts and thereby stimulating bone formation. Further studies are necessary to investigate the role of DKK1 in the prevention and treatment of osteoporosis.

The authors of this manuscript have certified that they comply with the Principles of Ethical Publishing in the International Journal of Cardiology [9].

\section{References}

[1] Hansson GK. Inflammation, atherosclerosis and coronary artery disease. N Engl J Med 2005;352:1685-95.
[2] Doherty TM, Fitzpatrick LA, Inoue D, Quiao JH, Fishbein MC, Detrano RC, et al. Molecular, endocrine and genetic mechanisms of arterial calcification. Endocr Rev 2004;25:629-72.

[3] Krishnan V, Bryant HU, MacDougald OA. Regulation of bone mass by Wnt signalling. J Clin Invest 2006;116:1202-9.

[4] Mani A, Radhakrishnan J, Wang HE, Mani A, Mani M, Nelson-Williams C, et al. LPR6 mutation in a family with early coronary disease and metabolic risk factors. Science 2007; 315:1278-82.

[5] Baron R, Rawadi G, Roman-Roman S. Wnt signalling: a key regulator of bone mass. Curr Topics Develop Biol 2006;76:103-27.

[6] Ueland T, Otterdal K, Lekva T, Sandberg W, Halvorsen B, Bollerslev J, et al. Increased circulating Dickkopf-1 in acute coronary syndrome. Association with platelet release and endothelial inflammatory response. J Bone Miner Res 2008;23:s282.

[7] Rajamannan NM, Subramaniam M, Caira F, Stock SR, Spelsberg TC. Atorvastatin inhibits hypercholesterolemia-induced calcification in the aortic valves via the Lrp5 receptor pathway. Circulation 2005;112(Suppl I):I229-34.

[8] Nusse R. Wnt signalling in disease and in development. Cell Research 2005;15:28-32.

[9] Coats AJ. Ethical authorship and publishing. Int J Cardiol 2009;131:149-50.

\title{
Impact of loading dose adjustment on platelet reactivity in homozygotes of the 2C19 2* loss of function polymorphism
}

\author{
Laurent Bonello ${ }^{\mathrm{a}, *}$, Nathalie Palot-Bonello ${ }^{\mathrm{b}}$, Sébastien Armero ${ }^{\mathrm{a}}$, Laurence Camoin-Jau ${ }^{\mathrm{c}}$, Franck Paganelli ${ }^{\mathrm{a}}$ \\ a Département de cardiologie, Hôpital Universitaire Nord, faculté de médecine, Marseille, France \\ b Département de génétique médicale, Hôpital de la Timone enfant, Marseille, France \\ c INSERM UMRS 608, Laboratoire d'hématologie, Hôpital de la Conception, Faculté de pharmacie, Marseille, France
}

A R T I C L E I N F O

\section{Article history:}

Received 9 July 2009

Accepted 25 July 2009

Available online 26 August 2009

\section{Keywords:}

Clopidogrel

Cytochrome polymorphism

Platelet reactivity monitoring

VASP index

Thrombosis

Platelet reactivity (PR) inhibition is critical to prevent recurrent thrombotic events in coronary artery disease (CAD) patients undergoing percutaneous coronary intervention $(\mathrm{PCI})$. It has been recently demonstrated that cytochromes (CYP) 2C19 2* loss of function polymorphism affects the level of PR inhibition induced by clopidogrel [1,2]. In fact this enzyme is critical for bio-transformation of clopidogrel into its active metabolites by contributing in the two sequential steps of activation. Mega et al. have observed that carriers of the mutant allele had reduced generation of clopidogrel active metabolites resulting in lower levels of PR inhibition and thus higher rates of post-PCI thrombotic events including stent thrombosis [2,3]. Homozygotes for the CYP 2C19 2* loss of function

\footnotetext{
* Corresponding author. Cardiology department, Hôpital nord, Chemin des bourrely, 13015 Marseille, France. Tel.: +33 491968858; fax: +33 491967989

E-mail address: laurentbonello@yahoo.fr (L. Bonello).
}

polymorphism may be at particularly high risk of events since the mutant allele results in the lack of enzymatic function [4]. Interestingly, we have recently demonstrated that increased or individually tailored loading dose (LD) of clopidogrel resulted in higher levels of PR inhibition in patients with high on-treatment platelet reactivity (HTPR) and a reduced thrombotic risk $[5,6]$. However the ability of such strategy to overcome HTPR in homozygote patients for CYP 2C19 2* polymorphism remains unknown. In the present study we aimed to investigate the ability of a strategy of individually tailored LD of clopidogrel to overcome HTPR in homozygotes for CYP 2C19 2* polymorphism.

We prospectively recruited 6 homozygotes for CYP 2C19 2* polymorphism who were scheduled for PCI. PR was measured using the VASodilator Phosphoprotein index (VASP index) in all patients at least $6 \mathrm{~h}$ after each LD of clopidogrel and before PCI and within $24 \mathrm{~h}$ of blood collection by an experienced investigator using Platelet VASP kits (Diagnostica Stago, Asnières, France) as previously described [5,6]. PR is expressed in this study as the VASP index corresponding to a ratio of the VASP phosphorylation of activated platelets versus at-rest platelets and expressed as a percentage of PR. LD adjustment was performed according to the previously described strategy. Briefly, patients with a VASP $<50 \%$ after the first clopidogrel LD were considered good responders (GR). Patients with a VASP $\geq 50 \%$ despite the $600 \mathrm{mg}$ LD were considered to have high on-treatment platelet reactivity (HTPR). For those patients clopidogrel pre-treatment was adjusted individually, before PCI, to obtain a VASP index $<50 \%$ using up-to 3 additional LD of $600 \mathrm{mg}$ each prescribed at $24 \mathrm{~h}$ intervals. The VASP index was assessed $12 \mathrm{~h}$ after each administration until a VASP index $<50 \%$ was obtained [3,4]. Genomic DNA was extracted from peripheral blood lymphocytes by standard 